\title{
Comparison of Beamforming Techniques for W-CDMA Communication Systems
}

\author{
Hsueh-Jyh Li and Ta-Yung Liu
}

\begin{abstract}
In this paper, different beamforming techniques are employed in the wide-band code-division multiple-access base station, and their uplink and downlink signal-to-(interference plus noise) ratio (SINR) performances are compared. It is found that the direction of arrival (DOA) method and the complex conjugate method almost have the same uplink SINR performance, but the complex conjugate method will shift the downlink main beam direction slightly due to the difference between the uplink and downlink carrier frequency. However, the degradation in the downlink mean SINR performance is less than $1 \mathrm{~dB}$ compared with that obtained by the DOA method. In the downlink, the SINR performances obtained by the single-beam method and multiple-beam beamforming technique are compared. It was found that the single-beam method has a poorer SINR performance in the low SINR region because it is more likely to suffer from deep fading. In the moderate or high SINR region, the single-beam method has a much better SINR performance because it has a higher gain in the main path direction and a smaller angular coverage of the mainlobe, which will result in a stronger signal level and smaller multiple-access interference at the mobile receiver.
\end{abstract}

Index Terms-Beamforming techniques, smart antennas, wideband code division multiple access (W-CDMA).

\section{INTRODUCTION}

$\mathbf{I}$ N THE code-division multiple-access (CDMA) system, multiple users use different code sequences to share the same frequency band at the same time. Due to the imperfect orthogonality among the different code sequences, multiple-access interference (MAI) is a major limitation to the channel capacity. Beamforming is a technique that can be used to focus the antenna beam to the desired user so that the signal-to-(interference plus noise) ratio (SINR) can be increased. RAKE combining is a technique that effectively utilizes the multipath energy so that the fading effect can be reduced and the signal power can be enhanced. Both beamforming and RAKE combining can effectively increase channel capacity.

In the wide-band CDMA (W-CDMA) system, an antenna array at the base station can be utilized to form beam patterns for the uplink and downlink. In the uplink, the received signal is first processed by a matched filter so that the chip positions (or time delays) of the dominant multipaths of the desired user can be determined. The antenna array is then used to determine the direction of arrival (DOA) of the multipath or to form a beam

Manuscript received December 15, 2000; revised September 25, 2001. This work was supported by the National Science Council, Republic of China, under Grant NSC 89-2219-E-002-018 and the MOE program for promoting academic excellence of universities under grant 89E-FA06-2-4-7.

The authors are with the Graduate Institute of Communication Engineering and the Department of Electrical Engineering, National Taiwan University, Taipei, Taiwan, R.O.C.

Digital Object Identifier 10.1109/TVT.2002.804864 pattern so that the desired signal can be constructively enhanced. In the downlink, the antenna array has to focus the beam to the desired user. However, because in the uplink and downlink, the carrier frequencies are different, and the fading effect can be uncorrelated in the short term, the beamforming effect can be different if the same weighting sets are used in both the uplink and downlink.

Several beamforming methods have been proposed and compared [1]-[4]. In particular, the complex conjugate method is based on the concept of retrodirectivity. It has the advantage that no additional computation loading is required in the uplink and downlink beamforming. However, the different carrier frequencies can degrade the downlink beamforming performance. To overcome this beamforming degradation, we can first determine the DOA of each dominant path of the desired user and then use the array manifold to calculate the required weighting factor for downlink. This method will increase the computation load and memory for signal processing. Since the fading effect may be uncorrelated for the uplink and downlink, it is interesting to compare the transmission performance when different beamforming algorithms are employed.

In this paper, we will compare the SINR performance obtained by different beamforming methods. We will apply a suitable propagation model and numerically calculate the SINR statistics for both uplink and downlink when different beamforming methods are used. In Section II, we will briefly describe an algorithm for channel parameter estimation and the twodimensional (2-D) RAKE receiver of the base station. In Section III, we will describe several beamforming methods and discuss their merits and drawbacks. In Section IV, we will use the desired propagation model to compare numerically the SINR statistics for different beamforming techniques. Discussion and conclusions will be given in Section V.

\section{Algorithm FOR CHANNEl PARAMETERS EstimAtion}

In the W-CDMA system proposed by ETSI [5], the known pilot bits can be used as the reference signals for channel parameter estimation. In the uplink, the data and pilot are separately binary phase-shift keying modulated on the I- and Q-channel, respectively. The binary data and pilot bits are spread using different orthogonal variable spreading factor codes to preserve orthogonality between the data and pilot channels. Different mobile users use different uplink scrambling codes to create a unique traffic channel. If we neglect the mutual interference between the data and pilot channels, the signal received at the base station can be separated into the I- and Q-channels. The $\mathrm{Q}$-channel is used to estimate the channel parameters. 


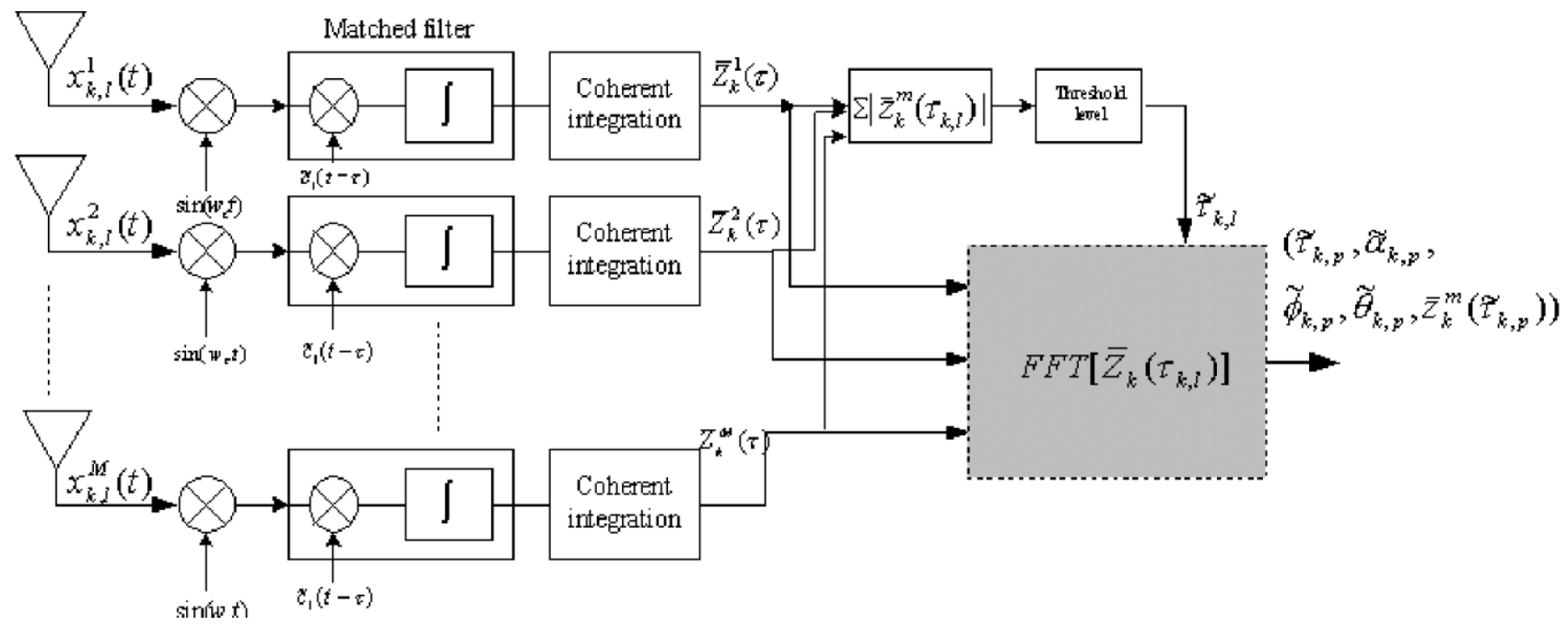

Fig. 1. Block diagram of the Q-channel for channel parameter estimation.

Assume that the channel is frequency selective. Signals transmitted from the users are received by an $M$-element antenna array for uplink signal reception. With perfect instantaneous power control, an equivalent complex baseband expression of the composite received signal vector $\mathbf{X}(t)$ at time $t$ is given by (1) at the bottom of the page and

$$
\sum_{l=1}^{L_{k}}\left(\alpha_{k, l}^{u}\right)^{2}=P_{r}, \quad \text { for all } K
$$

where it is assumed that there are $K$ users (one desired and $K-1$ interfering users). The $k$ th user has $L_{k}$ propagation paths. The parameters $\alpha_{k, l}^{u}, \phi_{k, l}, \tau_{k, l}$, and $\theta_{k, l}$ are, respectively, the uplink amplitude, phase shift, time delay, and angle of arrival of the $l$ th multipath component from the $k$ th user. $b_{k}(n)$ is the $n$th bit value, $c_{k}(t)$ is the spreading waveform assigned to the $k$ th user, and $T_{b}$ is the bit period. The column vector $\mathbf{a}_{k}\left(\theta_{k, l}\right)=$ $\left[1, a_{1}\left(\theta_{k, l}\right), \ldots, a_{M-1}\left(\theta_{k, l}\right)\right]^{T}$ is the array response vector corresponding to the path arriving on angle $\theta_{k, l}$, where $a_{m}\left(\theta_{k, l}\right)$ is a complex number denoting the amplitude gain and phase shift of the signal at the $(m+1)$ th antenna relative to that at the first an-

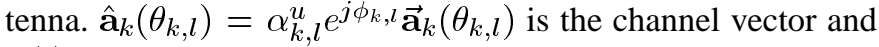
$\mathbf{n}(t)$ is the additive white Gaussian noise (AWGN) vector. $P_{r}$ is the desired total received power from the $k$ th user and is assumed to be constant for all $k$ because of perfect instantaneous power control.

Without loss of generality, assuming that the first user is the desired user and code synchronization has been established, the output of the matched filter (or called delay profile) can be written as

$\mathbf{z}_{1}(\tau)=T_{b} b_{1}(n) \sum_{l=1}^{L_{1}} \hat{\mathbf{a}}_{1}\left(\theta_{1, l}\right) \delta\left(\tau-\tau_{1, l}\right)+\mathbf{s}_{1}(\tau)+\mathbf{m}_{1}(\tau)+\eta_{1}(\tau)$

where $\mathbf{s}_{1}$ is the self-interference signal vector due to other multipath components of the desired user, $\mathbf{m}_{1}$ is the MAI vector, and $\eta_{\mathbf{1}}$ is due to the thermal noise vector. The output of the matched filter is used to distinguish the desired signal from the cochannel interference, and its time resolution is about the chip interval $T_{c}$. Despite the fact that the desired signal can be enhanced by a factor of the processing gain $T_{b} / T_{c}$, the most significant path components in the matched filter output may come from the undesired users. The larger the number of simultaneous users is, the poorer the received signal will be. The net interference severely limits the multiple-access capacity of the CDMA system. It was recognized that CDMA capacity is interference limited, and any reduction in interference converts directly and linearly into an increase in capacity [6]. To effectively reduce interference and provide accurate time bins for RAKE combining, the long scrambling code is adopted to average out the MAI signals. The block diagram of channel parameter estimation is shown in Fig. 1.

Assume that a time slot contains $N$ pilot bits and consider the slow fading multipath channels. The delay profile for each pilot bit (in one slot time) is first obtained. Under the assumption that the long scrambling code is used, the MAI for each pilot

$$
\begin{aligned}
\mathbf{x}(t) & =\sum_{k=1}^{K} \sum_{l=1}^{L_{k}} \alpha_{k, l}^{u} e^{j \phi_{k, l}} \sum_{n=-\infty}^{\infty} b_{k}(n) c_{k}\left(t-n T_{b}-\tau_{k, l}\right) \overrightarrow{\mathbf{a}}_{k}\left(\theta_{k, l}\right)+\mathbf{n}(t) \\
& =\sum_{k=1}^{K} \sum_{l=1}^{L_{k}} \hat{\mathbf{a}}_{k}\left(\theta_{k, l}\right) \sum_{n=-\infty}^{\infty} b_{k}(n) c_{k}\left(t-n T_{b}-\tau_{k, l}\right)+\mathbf{n}(t)
\end{aligned}
$$


bit can be modeled as independent Gaussian noise [7], [8]. It is also noted that during a time slot period $(0.625 \mathrm{~ms})$, the total phase change of the desired signal due to Doppler shift is small. Therefore the $N$ consecutive delay profile can be coherently integrated and averaged to obtain the mean delay profile. With this approach, the mean delay profile $\bar{z}_{1}^{m}$ at the $m$ th antenna element is given by

$$
\bar{z}_{1}^{m}(\tau)=\frac{1}{N} \sum_{n=1}^{N} z_{1, n}^{m}(\tau)
$$

where $z_{1, n}^{m}$ represents the matched filter output of the $n$th pilot bit for the $m$ th antenna element. It is noted that in the mean delay profile, for those time bins corresponding to the delay times of the desired user's multipath, their magnitudes will be increased by $N$ times after coherent integration. For all other bins, their magnitudes will be averaged out because the phases of the cross-correlation noise for different pilot bits are randomly distributed due to the characteristics of long scrambling code. In addition to coherent integration, the $M$ mean delay profiles can be exploited to further reduce the MAI signals and improve the multipath fading across the array elements. With this approach, we can noncoherently integrate the absolute value of the mean delay profile $\bar{z}_{1}^{m}$ over the $M$ elements as follows:

$$
\overline{\bar{z}}_{1}(\tau)=\frac{1}{M} \sum_{m=1}^{M}\left|\bar{z}_{1}^{m}(\tau)\right| .
$$

After noncoherent integration of (5), the fading effect across the array can be reduced substantially and the SINR can be improved significantly (9), (10). Then we can set a threshold and choose the desired time bins from the mean absolute delay profile of (5). The mean delay profile values of each array element at the selected time bins can be employed to calculate the beamforming weights for uplink and downlink. They can also be used to find the DOA of the desired multipaths. For example, by applying the Fourier transform to the mean delay profile with respect to the selected time bins, positions of the peaks give the estimation of the DOAs, and amplitudes of the peak values give the estimation of the path gains and phases of multipaths.

After the bin positions of the dominant paths of the desired user have been detected, the data signals in the I-channel are passed through 2-D RAKE correlators that are matched to the channel response of the transmitted waveform. The structure of the 2-D RAKE receiver is shown in Fig. 2. The signals received by each array element are first passed through $P$ correlators. Each correlator is matched to the desired user's code with a time shift equal to the time delay of the desired signal paths. These correlator outputs are then passed through a beamformer to further enhance the desired signal.

Several beamforming techniques have been proposed. Among these techniques, the complex conjugate method is the simplest one. It is based on the concept of retrodirectivity. The method is described as follows.

1) In the Q-channel, we have obtained the mean delay profile of each element, which is calculated by (5). Denote the value sampled at the estimated delay time $\tilde{\tau}_{1, p}$ by $\bar{z}_{1}^{m}\left(\tilde{\tau}_{1, p}\right)$.
2) The correlator output of the $m$ th element in the I-channel is then multiplied by the complex conjugate of $\bar{z}_{1}^{m}\left(\tilde{\tau}_{1, p}\right)$.

3) Coherently integrate all the $M$ multiplication values. This is the output of the $p$ th beamformer.

4) The RAKE combiner then coherently combines the $P$ beamformer outputs.

The beamforming can also be obtained by multiplying the correlator output of each element by an array factor formed by the DOA of the desired path. The DOA of the desired path can be obtained by applying the Fourier transform to the sample value $\bar{z}_{1}^{m}\left(\tilde{\tau}_{1, p}\right)$ in the Q-channel. Denote the estimated DOA and amplitude of the $p$ th path as $\tilde{\theta}_{1, p}$ and $\hat{\alpha}_{1, p}=\tilde{\alpha}_{1, p} e^{-j \tilde{\phi}_{1, p}}$, respectively. Then the $p$-th correlator output is multiplied by $\mathbf{a}_{1}^{m^{*}}=e^{j 2 \pi(m-1) d \cos \tilde{\theta}_{1, p} / \lambda}$, where $d$ is the spacing between antenna elements. With this method, the antenna array will form a beam to the DOA of the desired signal. In the RAKE combiner, the $p$ th correlator output will multiply $\hat{\alpha}_{1, p}^{*}$, the complex conjugate of $\hat{\alpha}_{1, p}$, and then be coherently integrated. It is noted that the DOA information can have several applications. For example, it can be used for the downlink beamforming. It can also be used to locate the mobile location [11], [12].

Other adaptive beamforming algorithms have been proposed to null the interference [11]. However, it is known that the maximum number of interferers that can be nulled is $M-1$ if $M$ antenna elements are employed. In the W-CDMA environment, the number of interferers in general exceeds the number of array elements. Therefore we do not attempt to null the interferers.

After multipath parameters of the desired signals have been estimated, these data can be fed into the data channel to evaluate the SINR of the 2-D RAKE receiver. The expected SINR of the 2-D RAKE receiver can be written as

$$
\mathrm{SINR}=\frac{E\{S(n)\}}{E\{I(n)\}}=\frac{M G E\left\{\left|\sum_{p=1}^{P} \mathbf{w}_{1, p}^{*} \mathbf{S}_{1, p}(t)\right|^{2}\right\}}{E\left\{\left|\sum_{p=1}^{P} \mathbf{w}_{1, p}^{*} \mathbf{U}_{1, p}(t)\right|\right\}}
$$

where $M$ is the number of antenna elements, $G$ is the processing gain, $P$ is the number of fingers of the RAKE receiver, $\mathbf{W}_{1, p}$ represents the array weighting vectors, $\mathbf{S}_{1, p}=\alpha_{1, p}^{u} e^{j \phi_{1, p}} \overrightarrow{\mathbf{a}}\left(\theta_{1, p}\right) b_{1}(n)$, and $\mathbf{U}_{1, p}(t)$ is the summation of all undesired signals plus thermal noise at the $p$ th finger of the RAKE receiver. If we assume that the system has a perfect power control so that all users have the same power level and the MAI and noise are both temporally and spatially white, then the SINR after the 2-D RAKE receiver can be approximated by [13]

$$
\operatorname{SINR}=\frac{\mathrm{GM}(\mathrm{SNR})}{(K-1) \mathrm{SNR}+1}
$$

where SNR is the uplink signal-to-noise ratio for each antenna element and $K$ is the number of active users.

\section{DOWNLINK BEAMFORMING AND SignAl PROCESSING}

In the W-CDMA/frequency-division duplex (FDD) system, there is more than $190-\mathrm{MHz}$ difference between the uplink 


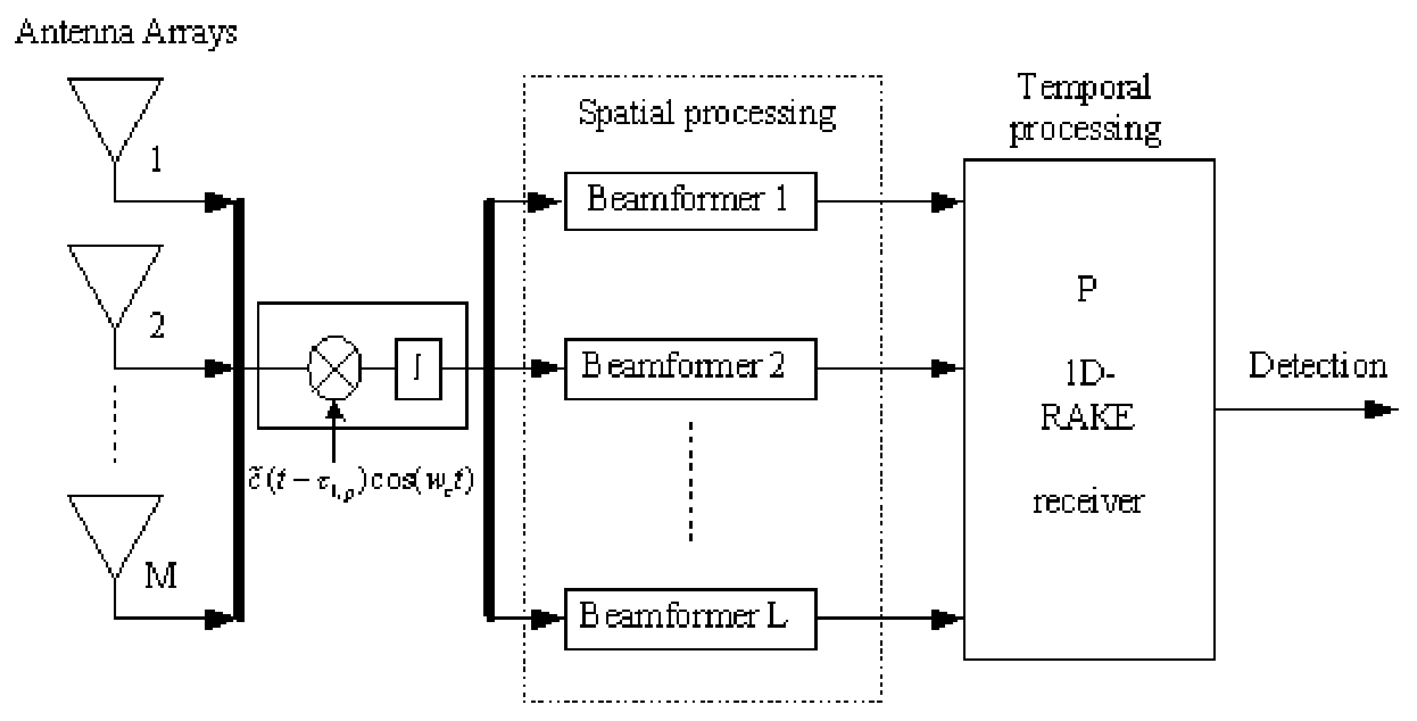

(a)

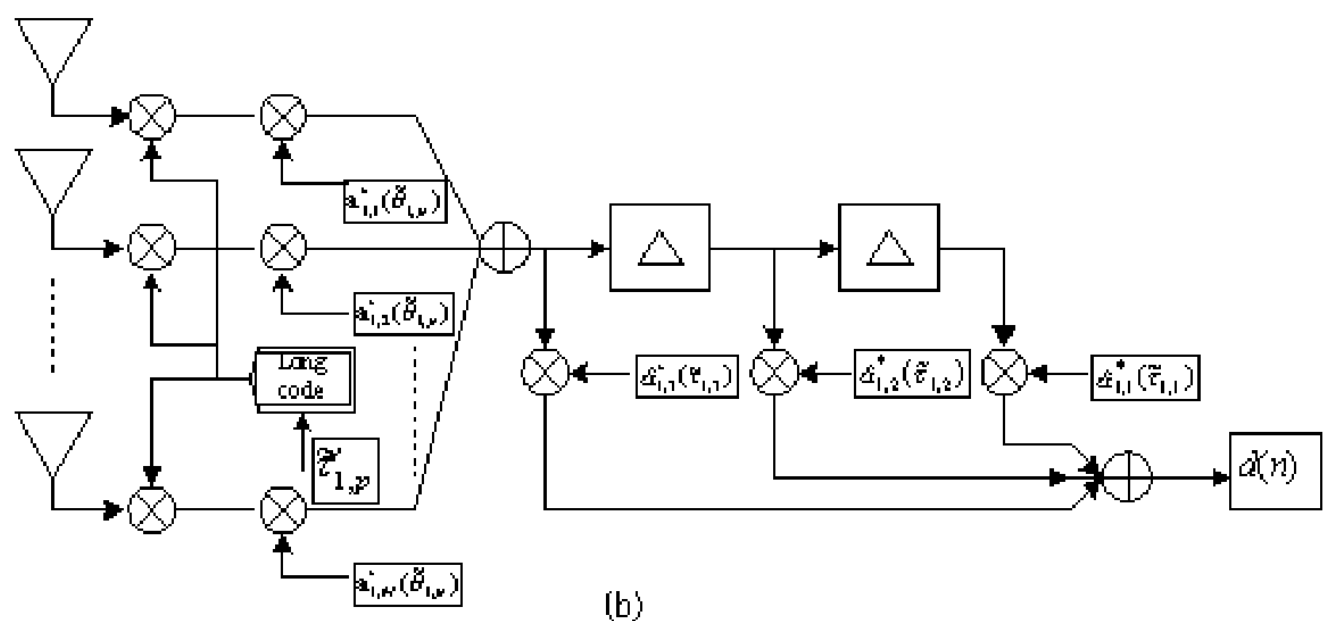

Fig. 2. Block diagram of the 2-D RAKE receiver: (a) coarse block diagram of spatial and temperal processing and (b) detail block diagram of beamforming and RAKE combining.

and downlink carrier frequencies. Measurement studies have indicated that multipath fading can be very sensitive to the carrier frequency. In downlink beamforming, several factors can be considered. For example, how will the performance be changed if the same element weightings as used in the uplink beamforming are used in the downlink? Should the downlink pattern be beamformed to a single dominant direction or to several multipath directions? If multiple beams were used, how will the energy ratio among different beams be decided? In the following, we will discuss several downlink beamforming algorithms. We will consider the problem from several points of view, such as the complexity of implementation, the effect of fading, and the multiple-access interference. In the downlink, denote the weighting of the $m$ th element for the desired user as $w_{m}$. To evaluate the downlink SINR performance, we further made the following assumptions.

1) The reciprocity property holds for the uplink and downlink. Therefore, the uplink and downlink will encounter the same multipath. However, the amplitudes of each multipath component can be different due to different carrier frequencies and different radiation patterns of the uplink and downlink.

2) Only the intracell interference is considered.

3) The base station transmits the same power for each coding channel.

The beamforming algorithms to be considered are the complex conjugate method and the DOA method. The types of beams to be synthesized are the single-beam and multiple-beam types. The weightings of different combinations are described as follows.

\section{A. The Single-Beam Complex Conjugate Method}

In the uplink, from the mean delay profile obtained by (5), choose the time bin with the largest peak value. Denote it by $\tau_{\max }$. The matched filter output of the $m$ th element sampled at 
$\tau_{\max }$ is denoted by $\bar{z}_{1}^{m}\left(\tau_{\max }\right)$. For the downlink, the weighting of each element is given by

$$
w_{m}=\bar{z}_{1}^{m^{*}}\left(\tau_{\max }\right) .
$$

\section{B. The Multiple-Beam Complex Conjugate Method}

From the uplink mean absolute delay profile of (5), choose the $P$ peak positions with the first $P$ largest values and denote these peak positions as $\tau_{1}, \ldots, \tau_{P}$. Denote the matched filter output of the $m$ th element sampled at $\tau_{1}, \ldots, \tau_{P}$ as $\bar{z}_{1}^{m}\left(\tau_{1}\right), \ldots, \bar{z}_{1}^{m}\left(\tau_{P}\right)$. The weighting of the $m$ th element is then given by

$$
w_{m}=\sum_{p=1}^{P} \bar{z}_{1}^{m^{*}}\left(\tau_{p}\right) \text {. }
$$

\section{The Single-Beam DOA Method}

Denote the DOA of the dominant path $\tau_{\max }$ as $\theta_{\max }$. The weighting of the $m$ th element is given by

$$
w_{m}=e^{j k(m-1) d \cos \left(\theta_{\max }\right)}
$$

where $k$ is the wavenumber at the downlink carrier frequency.

\section{The Equal-Gain Multiple-Beam DOA Method}

Suppose that the estimated DOAs of the dominant paths of the desired user are $\tilde{\theta}_{1}, \ldots, \tilde{\theta}_{P}$. The weighting of the $m$ th element is given by

$$
w_{m}=\sum_{p=1}^{P} e^{j k(m-1) d \cos \left(\tilde{\theta}_{p}\right)} .
$$

\section{E. The Maximal-Ratio Multiple-Beam DOA Method}

Suppose that the estimated DOAs of the dominant paths and their corresponding magnitudes are $\tilde{\theta}_{1}, \ldots, \tilde{\theta}_{P}$ and $\tilde{m}_{1}, \ldots, \tilde{m}_{P}$, respectively. Then the weighting of the $m$ th element is given by

$$
w_{m}=\sum_{p=1}^{P} \tilde{m}_{p} e^{j k(m-1) d \cos \left(\tilde{\theta}_{p}\right)} .
$$

To have a fair comparison, we constrain that the total radiated power to be constant for different beamforming algorithms. Therefore, each element weight should be normalized by

$$
\tilde{w}_{m}=\frac{w_{m}}{\left(\sum_{m=1}^{M}\left|w_{m}\right|^{2}\right)^{1 / 2}} .
$$

If the channel were stationary, it would be better to have a single beam because the antenna pattern will be focused to the direction with the maximum transmission. However, as the uplink and downlink fading are independent due to the different carrier frequencies, the use of a single-beam pattern will be more likely to suffer from deep fading than the multiple-beam pattern. The relation between the fourth and the fifth methods is equivalent to that of the equal-gain and maximum-ratio gain combining in the RAKE combiner.
TABLE I

RADIO LINK PARAMETERS OF THE SIMULATIONS

\begin{tabular}{|c||c}
\hline $\begin{array}{c}\text { Chip rate } \\
\text { (Bandwridth) }\end{array}$ & $4.096 \mathrm{Mcps}$ (5MHz) \\
\hline $\begin{array}{c}\text { Cartier frequency } \\
\text { (Uplink/Downtlink) }\end{array}$ & $1.965 \mathrm{GHz} / 2.155 \mathrm{GHz}$ \\
\hline $\begin{array}{c}\text { Pilot bits/slot } \\
\text { (Uplink/Downlink) }\end{array}$ & 6 bits/4bits \\
\hline Bit rates & $16 \mathrm{Kbps}$ \\
\hline Processing gain & 256 \\
\hline Spreading code & Truncated-G old sequence $\left(40960 \times 2^{41}\right)$ \\
\hline Modulation & BPSK \\
\hline Slot time & $0.625 \mathrm{~ms}$ \\
\hline
\end{tabular}

TABLE II

Channel Parameters of THE Desired User (Without FADING)

\begin{tabular}{|c||c|c|c||}
\hline $\begin{array}{c}\text { PATH } \\
\text { \# }\end{array}$ & 1 & 2 & 3 \\
\hline$\theta_{11}$ & $-40^{\circ}$ & $0^{\circ}$ & $40^{\circ}$ \\
\hline$\tau_{11}$ & 0 & $4 T_{\mathrm{r}}$ & $8 T_{\mathrm{r}}$ \\
\hline $\boldsymbol{a}_{1 \mathbf{1}}$ & 1 & 0.65 & 0.8 \\
\hline $\begin{array}{c}\phi_{11} \\
\text { (rad) }\end{array}$ & 3.1416 & 1.5708 & 4.7124 \\
\hline
\end{tabular}

From the interference point of view, it is reasonable to assume that the DOA distribution of the undesired users is uniform if the number of users is large (for example, more than 30). It is known that the beamwidth is proportional to the antenna size or number of elements. If a single beam is used, the number of users to be interfered within the mainlobe coverage will be smaller than that when a multiple-beam is used, because the multiple-beam pattern will have a wider angular coverage.

In the next section we will use a propagation model to calculate numerically the SINR statistics when different beamforming techniques are employed in the uplink and downlink.

\section{Simulation Results}

In this section, we compare the uplink and downlink SINR performance obtained by different beamforming algorithms.

Assume that in the control channel, a slot time $(0.625 \mathrm{~ms})$ contains six pilot bits in the uplink and four pilot bits in the downlink and these pilot bits are used to estimate the channel parameters. Consider a uniformly spaced linear array with seven antenna elements and $d=\lambda_{u} / 2$ spacing, where $\lambda_{u}$ is the uplink wavelength. The major uplink and downlink radio parameters of the simulations are listed in Table I. Assume that the SNR for each antenna element is $10 \mathrm{~dB}$. The channel parameters about the desired user is shown in Table II. There are three paths, and the DOAs are $-40^{\circ}, 0^{\circ}$, and $40^{\circ}$, respectively. The relative time delays are $0,4 T_{c}$, and $8 T_{c}$, respectively, where $T_{c}$ is the chip duration. The relative path amplitudes are $1,0.65$, and 0.8 respectively. The phases are $\pi, \pi / 2$, and $3 \pi / 2$, respectively. Assume that there are 40 active cochannel users and each user 


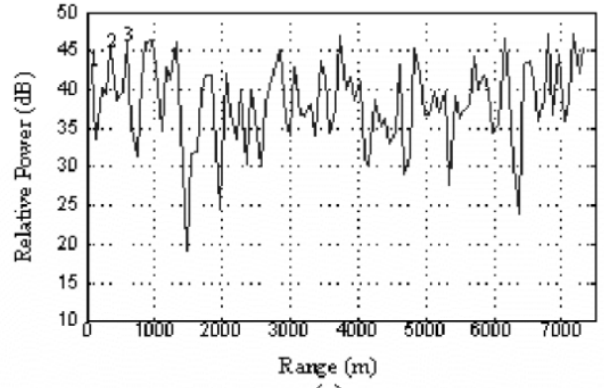

(a)

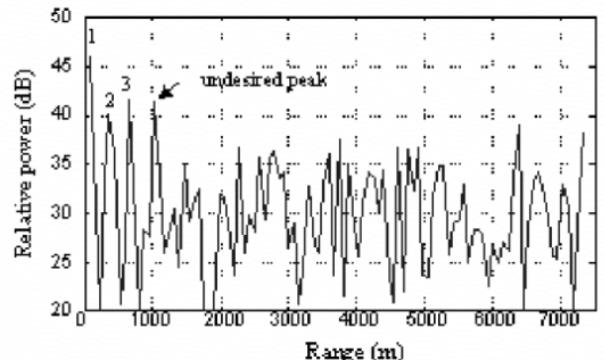

(b)

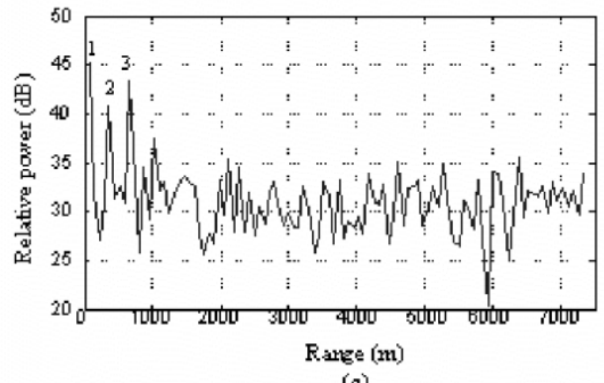

(c)

Fig. 3. Matched filter output (power delay profile): (a) without coherent integration, (b) with coherent integration over six pilot bits, (c) with coherent integration over six pilot bits and incoherent integration over seven antenna elements.

also has three multipaths. Assume that the distributions of the channel parameters $\alpha_{k, l}, \phi_{k, l}$, and $\tau_{k, l}$ for the undesired users are Rayleigh, uniform over $[0, \pi / 2]$, and uniform over $\left[0, \tau_{\max }^{\prime}\right]$, respectively, where $\tau_{\max }^{\prime}$ is the maximum delay spread of the channel. Also assume that the DOA $\theta_{k, l}$ of the undesired users is uniformly distributed over $[-\pi / 3, \pi / 3]$.

Fig. 3(a) shows an example of the output of the matched filter of an antenna element, which is obtained by using only one pilot bit. It is seen that there are many peaks and we cannot distinguish the desired signal peaks from the interfering signal peaks. If we coherently integrate the matched filter outputs over six pilot bits, the result is shown in Fig. 3(b). One can easily find that the values of the desired paths (noted by 1,2, 3 in the figure) have been substantially enhanced. However, there is an undesired peak that is greater than the desired peak, which will give a wrong time of arrival for RAKE combining. If we further incoherently integrate the mean delay profiles over seven antenna elements, the result is shown in Fig. 3(c). It is noted that the undesired peak has been reduced and the desired paths can be correctly identified. Denote these times of arrival as $\tilde{\tau}_{1, p}$.

Next we compare the patterns formed by the complex conjugate method and the DOA method. At each estimated

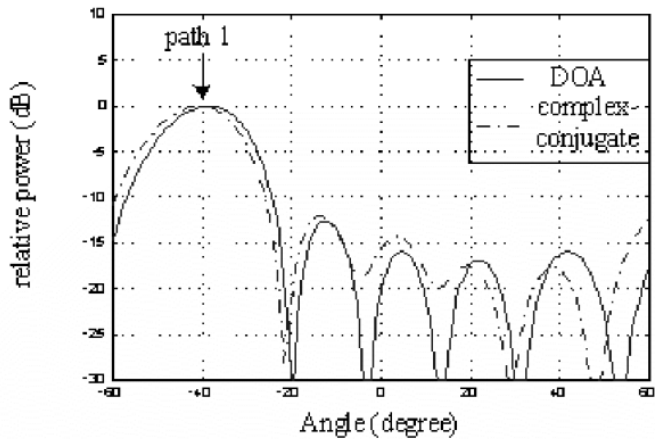

(a)

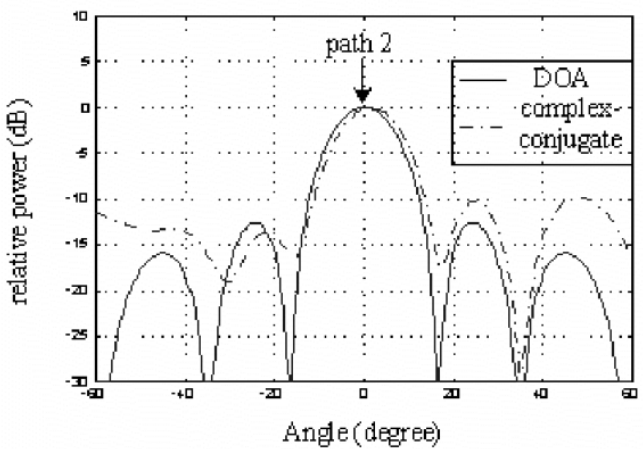

(b)

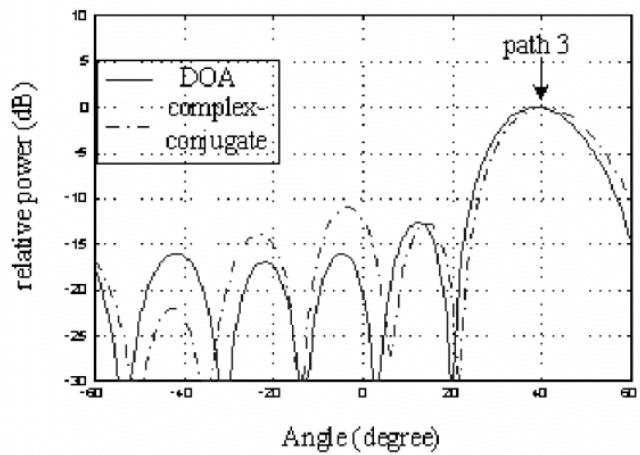

(c)

Fig. 4. Uplink antenna patterns synthesized by the DOA and complex-conjugate method: (a) Path 1, (b) path 2, (c) path 3.

$\tilde{\tau}_{1, p}$, we obtain the $\bar{z}_{1}^{m}\left(\tilde{\tau}_{1, p}\right)$ for each element. The pattern formed by the complex conjugate method can be expressed by $\sum_{m=1}^{M} \bar{z}_{1}^{m^{*}}\left(\tilde{\tau}_{1, p}\right) \cdot a_{m}(\theta)$, where $a_{m}(\theta)=e^{j k(m-1) d \cos (\theta)}$. This pattern is the Fourier transform (FT) of $\overline{\mathbf{z}}_{1}^{*}\left(\tilde{\tau}_{1, p}\right)$ with respect to $m$. By the DOA method, we apply the FT to the sampled value $\bar{z}_{1}^{m}\left(\tilde{\tau}_{1, p}\right)$ and obtain the DOA $\tilde{\theta}_{1, p}$. The beam pattern formed by the DOA method can then be expressed by $\overrightarrow{\mathbf{a}}^{*}\left(\tilde{\theta}_{1, p}\right) \cdot \overrightarrow{\mathbf{a}}(\theta)$. The resultant patterns obtained by the above two methods are shown in Fig. 4(a) to (c), respectively, for $p=1,2,3$. In fact, the patterns formed by the complex conjugate method and the DOA method should be identical if the SINR is significantly high. However, the pattern formed by the complex conjugate method will be degraded if the MAI is increased. One can easily find that in Fig. 4, patterns obtained by both methods have almost the same mainlobe level, but the patterns obtained by the complex conjugate method (dashed curves) have higher sidelobe levels.

Next we demonstrate the downlink patterns formed by the five beamforming techniques stated in the previous section. Use 


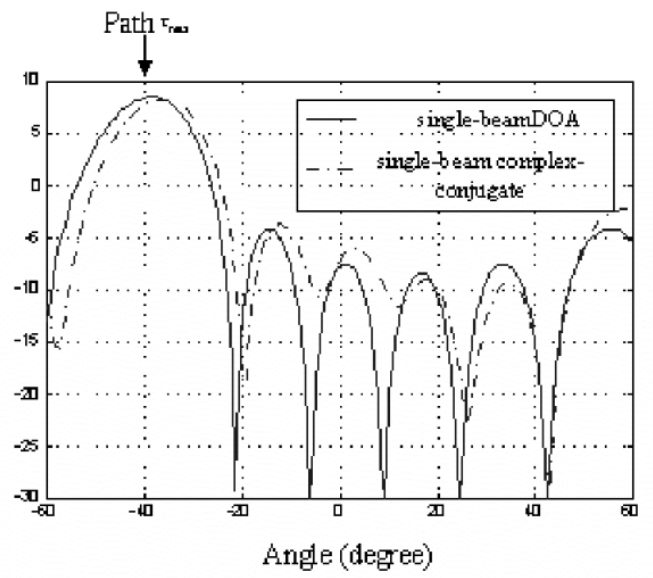

(a)

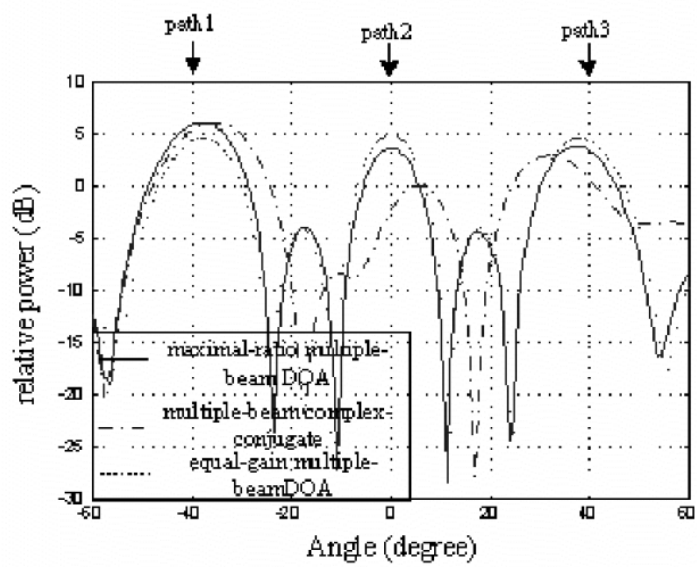

(b)

Fig. 5. Downlink patterns synthesized by different beamforming techniques: (a) by single-beam DOA method and single-beam complex conjugate method; (b) by maximal-ratio multiple-beam DOA method with maximal-ratio combining and equal-gain combining, and multiple-beam complex conjugate method.

the same parameter sets of the above example. The five downlink-synthesized patterns are shown in Fig. 5(a) and (b). It is seen that the mainlobe directions obtained by the complex conjugate method have shifted a small value due to the difference between the uplink and downlink carrier frequencies. The pattern difference between the complex conjugate method and the DOA method is more pronounced in the multiple-beam case than in the single-beam case.

Neglect the uplink and downlink fading effect. Assume that the channel parameters of the desired user and the parameter distributions of the undesired users are the same as in the previous example. We conducted 200 independent interference simulations for each fixed number of active users. We first calculate the uplink SINR after RAKE combiner using the two-uplink beamforming techniques for each parameter set and then calculate the mean SINR averaged over the 200 simulations. The resultant mean SINR versus the number of interfering users is shown in Fig. 6. The theoretical values [obtained by (7)] are also shown in the figure for comparison. It is seen that both methods almost have the same performance and approach the theoretical values. This result can be explained by observing the synthesized antenna patterns of Fig. 4. Although the two patterns have

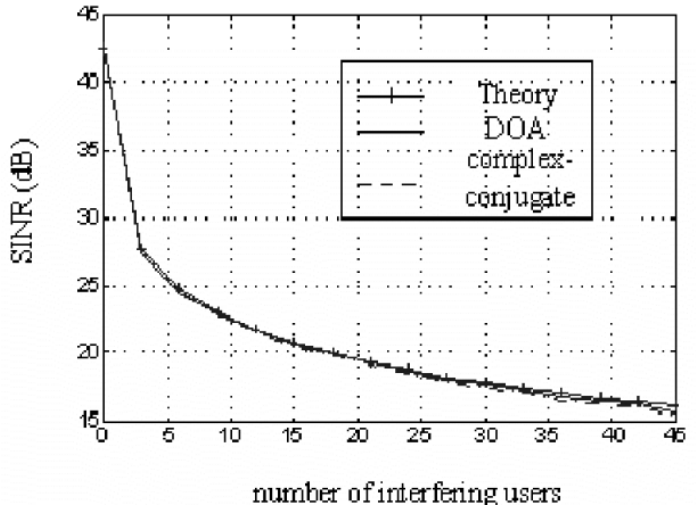

Fig. 6. Plots of the uplink mean SINR performance versus number of interferers.

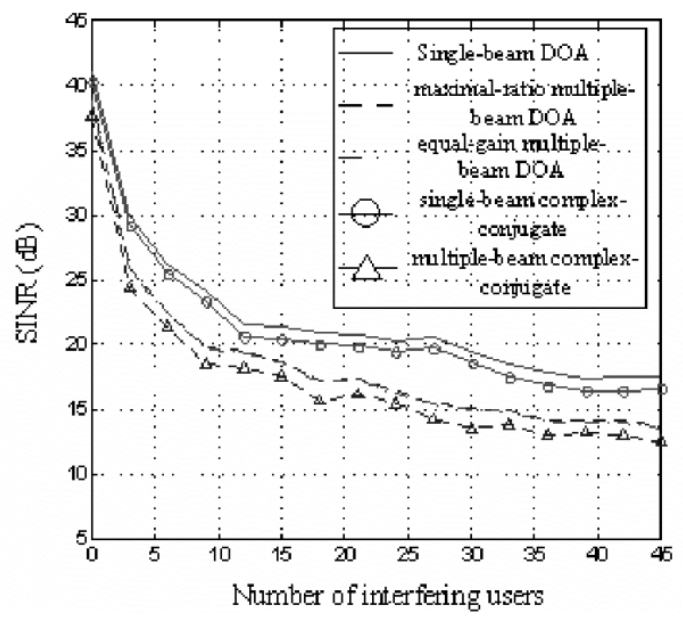

Fig. 7. Plots of the downlink mean SINR versus number of interferers when different downlink beamforming methods are used.

different sidelobe levels, they have almost the same mainlobe level. The sidelobes have little contribution to the SINR when the SINR is high (for example, greater than $10 \mathrm{~dB}$ ). Therefore the resultant SINRs for both methods are almost the same.

Next we compare the downlink SINR performance obtained by the five different beamforming techniques. We assume that all users use the same beamforming techniques. We use the measured matched filter output and the derived DOA at the uplink and then apply the five downlink beamforming techniques to calculate the field, including the desired signal and interference, received at the mobile. Assume that a time slot contains four pilot bits in the downlink. For each parameter set, we calculate the downlink SINR after RAKE combiner. The mean SINRs obtained by the five techniques are shown in Fig. 7. It is seen that the single-beam method has a much better performance than the multiple-beam method. Moreover, the DOA method gives a better performance than the complex conjugate method. This result can also be explained by observing the synthesized patterns of Fig. 5. It is noted that the single-beam method has a higher gain in the desired path direction and smaller total angular coverage in the mainlobe. This will give a higher desired signal level and a smaller MAI in the mobile receiver.

Next we consider the fading effect of each multipath. The channel parameters of the desired user are shown in Table III. 
TABLE III

Channel Parameters of the Desired User (With Fading)

\begin{tabular}{|c||c|c|c|c|c|c|}
\hline \hline CLUSTER & \multicolumn{2}{|c|}{1} & \multicolumn{2}{c|}{2} & \multicolumn{2}{c|}{3} \\
\hline PATH & 1 & 2 & 3 & 4 & 5 & 6 \\
\hline $\begin{array}{c}\theta_{11} \\
\left( \pm^{0}\right)\end{array}$ & $\begin{array}{c}0^{0} \\
\left( \pm 5^{0}\right)\end{array}$ & $\begin{array}{c}0^{0} \\
\left( \pm^{0}\right)\end{array}$ & $\begin{array}{c}-40^{0} \\
\left( \pm 5^{0}\right)\end{array}$ & $\begin{array}{c}-40^{0} \\
\left( \pm^{0}\right)\end{array}$ & $\begin{array}{c}40^{0} \\
\left( \pm 5^{0}\right)\end{array}$ & $\begin{array}{c}40^{0} \\
\left( \pm 5^{0}\right)\end{array}$ \\
\hline$\tau_{11}$ & 0 & $2 T_{c}$ & $4 T_{c}$ & $7 T_{c}$ & $8 T_{c}$ & $9 T_{c}$ \\
\hline fading & Rayleigh & Rayleigh & Rayleigh & Rayleigh & Rayleigh & Rayleigh \\
\hline \hline
\end{tabular}

The desired user has three clusters of multipaths. Each cluster contains two paths. The mean DOAs of these three clusters are $-40^{\circ}, 0^{\circ}$, and $40^{\circ}$, respectively, and each DOA of the multipath is uniformly distributed over $\left[-5^{\circ}, 5^{\circ}\right]$ about the respective mean DOA. Assume that the distance between the base station and the desired mobile station is $1.4 \mathrm{Km}$ and that the relative time delays of multipath components are shown in Table III. We also assume that each cochannel user has six paths and that the DOAs of each path are uniformly distributed over $[-\pi / 3, \pi / 3]$. The instantaneous amplitude of each path has a Rayleigh fading. The uplink and downlink have independent Rayleigh fading for the FDD system considered in the simulation. The phase shifts of each path are uniformly distributed over $[0,2 \pi]$.

For each of the three cases of 1,20, and 40 total active users, we randomly generate 1000 parameter sets for each case. For each parameter set, we calculate the fields received by each antenna element and find the required weightings for the downlink using the five beamforming techniques. For the downlink, we assume that the base station transmits equal power for each user channel. Using the propagation model described in the previous paragraph, we calculate the downlink SINR after the mobile station's RAKE combiner. For the three different numbers of active users, we count the cumulative distribution function of the SINR for five different beamforming techniques. The SINR statistics are shown in Fig. 8(a) and (b), respectively.

Fig. 8(a) compares the performance obtained by the single-beam method and the multiple-beam method. It is seen that in the low SINR region, the multiple-beam method has a better SINR performance because the probability that all paths having deep fading is much smaller. In the high SINR region, the single-beam method has a better SINR performance because at the mobile receiver it has a stronger desired signal and a smaller interference, as explained in the previous example. Fig. 8(b) compares the SINR performance obtained by the complex conjugate method, the DOA method with equal gain, and the DOA method with maximal ratio, all using the multiple-beam beamforming technique. It is seen that the DOA method has a better performance than the complex conjugate method. The maximal-ratio method has a slightly better performance than the equal-gain method, but the difference is very small.

\section{DISCUSSION AND CONCLUSION}

In this paper, we study and compare the uplink and downlink SINR performance when different beamforming techniques are applied in the antenna array of the base station. In the up-

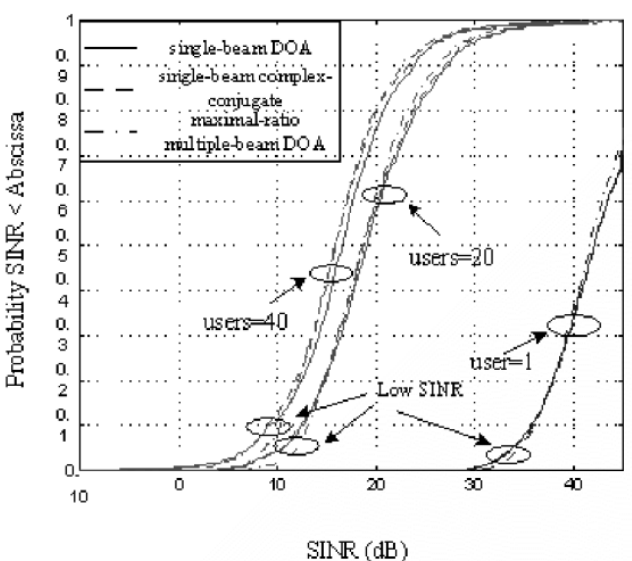

(a)

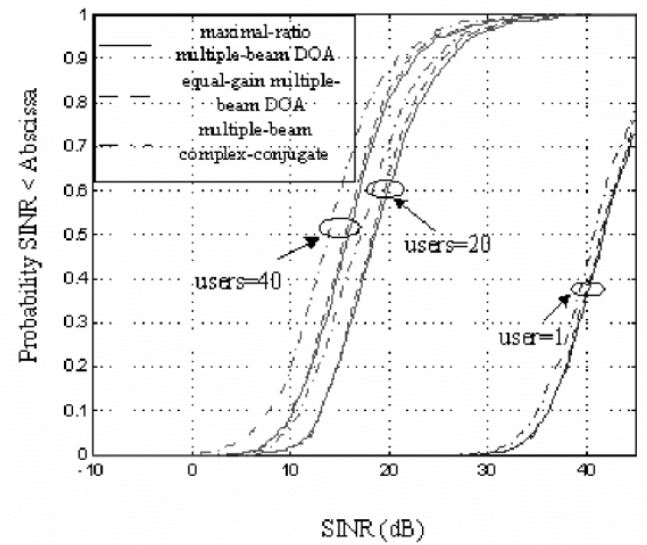

(b)

Fig. 8. Plots of the downlink SINR statistics obtained by different beamforming methods: (a) by single-beam DOA method, single-beam complex conjugate method, and multiple-beam maximal-ratio combining method; (b) by multiple-beam maximal-ratio combining method, multiple-beam equal-gain combining method and multiple-beam complex conjugate method.

link, we compare the DOA method and the complex conjugate method. We found that these two methods have almost the same SINR performance. However, the complex conjugate method does not need any additional computation loading. In the downlink, we compare the single-beam and multiple-beam beamforming techniques. We found that the multiple-beam method has a better SINR performance in the low SINR region because the single-beam method is more likely to suffer from deep fading. In the moderate or high SINR region, the single-beam method has a much better SINR performance because it has a higher antenna gain in the main path direction and a smaller angular coverage of the mainlobe, which will result in a stronger signal level and smaller MAI at the mobile receiver. Furthermore, it can reduce the delay spread of multipaths. The complex conjugate method will shift the downlink mainlobe slightly due to the difference in carrier frequencies between the uplink and the downlink. This will cause SINR degradation. However, the degradation is smaller than $1 \mathrm{~dB}$. Therefore, the complex conjugate method is a valuable method if the DOA information is not needed for further applications. We also found that the DOA method with maximal-ratio gain has a slightly better SINR performance than that with the equal-gain beamforming. However, the difference is very small. 


\section{REFERENCES}

[1] H. Asakura and T. Matsumoto, "Cooperative signal reception and downlink beam forming in cellular mobile communications," IEEE Trans. Veh. Technol., vol. 48, pp. 333-341, Mar. 1999.

[2] S. S. Jeng, G. T. Okamoto, G. Xu, H. P. Lin, and W. J. Vogel, "Experimental evaluation of smart antenna system performance for wireless communications," IEEE Trans. Antennas Propagat., vol. 46, pp. 749-757, June 1998.

[3] J. Ylitalo and M. Katz, "An adaptive antenna method for improving downlink performance of CDMA base station," in Proc. ISSSTA'98, 1998, pp. 599-603.

[4] G. V. Tsoulos, G. E. Athanasiadou, and R. J. Piechocki, "Low-complexity smart antenna methods for third generation W-CDMA systems," IEEE Trans. Veh. Technol., vol. 49, no. 6, pp. 2382-2396, 2000.

[5] "Spreading and modulation (FDD)," 3rd Generation Partnership Project (3GPP), TS 25.213 V2.0.0, 1999-4.

[6] A. J. Viterbi, "The orthogonal-random waveform dichotomy for digital mobile personal communication," IEEE Personal Commun., pp. 18-24, 1994.

[7] D. J. Torrieri, "Performance of direct-sequence system with long pseudonoise sequence," IEEE J. Select. Areas Commun., vol. 10, pp. 770-781, May 1992.

[8] G. L. Turin, "The effects of multipath and fading on the performance of direct-sequence CDMA system," IEEE Trans. Veh. Technol., vol. VT-33, pp. 213-219, Aug. 1984.

[9] T. Y. Liu, "Spatial- and time-domain signal processing for W-CDMA communication system," Ph.D. dissertation, Department of Electrical Engineering, National Taiwan University, 2001.

[10] M. H. Kao, "Smart antenna for W-CDMA systems," Master, Graduate Institute of Communication Engineering, National Taiwan University, 2001.

[11] J. C. Liberti and T. S. Rappaport, Smart Antenna for Wireless Communications IS-95 and 3rd Generation CDMA Applications. Englewood Cliffs, NJ: Prentice-Hall, 1999.

[12] I. Jami, M. Ali, and R. F. Ormondroyd, "Comparison of methods of locating and tracking cellular mobiles," Novel Methods of Location and Tracking of Cellular Mobiles and their System Applications (Ref. 1999/046), pp. 1/1-1/6, 1999.
[13] J. E. Hudson, Adaptive Array Principles. Stevenage, U.K.: Peter Peregrinus, 1989.

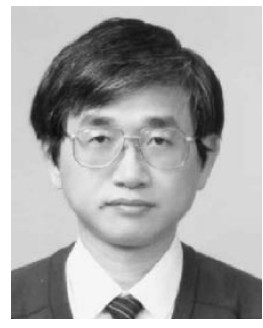

Hsueh-Jyh Li was born in Yun-Lin, Taiwan, R.O.C., on August 11, 1949. He received the B.S.E.E. degree form National Taiwan University, Taiwan, in 1971 and the M.S.E.E. and Ph.D. degrees from the University of Pennsylvania, Philadelphia, in 1980 and 1987, respectively.

Since 1973, he has been with the Department of Electrical Engineering, National Taiwan University, where he is a Professor. He was with the ElectroOptics and Microwave-Optics Laboratory of the University of Pennsylvania during 1984-1987. He was the Director of Communication Research Center during 1996-2000 and the Chairman of the Graduate Institute of Communication Engineering of National Taiwan University during 1997-2000. His main research interests are in microstrip antennas, radar scattering, microwave imaging, radio channel characteristics, and wireless communication.

Prof. Li received the Distinguished Research Award from the National Science Council, R.O.C., in 1992.

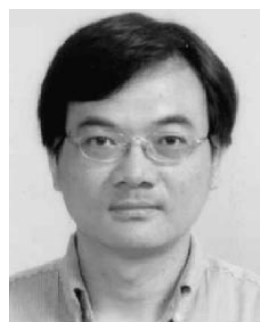

Ta-Yung Liu was born in Chia Yi, Taiwan, R.O.C., on April 22, 1961. He received the B.S.E.E. degree from Chung Cheng Institute of Technology in 1983 and the M.S.E.E. and Ph.D. degrees from the Department of Electrical Engineering, National Taiwan University, in 1989 and 2001, respectively.

He was with the Chung Shan Institute of Technology, R.O.C., from July 1983 to September 1996. $\mathrm{He}$ is now with Via Technologies, Inc. His research interests are in the areas of microwave imaging and digital radio. 